\title{
Synthesis and Crystal Structure of N-(2-Pyridylmethyl)-L-Alanine) Isothiocyanate Cobalt(III)
}

\author{
Sally-Judith E. Ntum¹, Awawou G Paboudam1, Asseng M. Conde², Linda D. Nyamen1, \\ Aminou Mohamadou ${ }^{3}$, James Raftery ${ }^{4}$, Peter T. Ndifon ${ }^{*}$ \\ ${ }^{1}$ Department of Inorganic Chemistry, Faculty of Science, University of Yaoundé 1, Yaoundé, Cameroon \\ ${ }^{2}$ Department of Chemistry, Faculty of Science, University of Douala, Douala, Cameroon \\ ${ }^{3}$ Institut de Chimie Moléculaire de Reims (ICMR), Groupe de Chimie de Coordination, Université de Reims \\ Champagne-Ardenne, Reims, France \\ ${ }^{4}$ School of Chemistry and Materials Science, University of Manchester, Manchester, UK \\ Email: *pndifon@yahoo.com
}

How to cite this paper: Ntum, S.-J.E., Paboudam, A.G., Conde, A.M., Nyamen, L.D., Mohamadou, A., Raftery, J. and Ndifon, P.T. (2017) Synthesis and Crystal Structure of $\mathrm{N}$-(2-Pyridylmethyl)-L-Alanine) Isothiocyanate Cobalt(III). Crystal Structure Theory and Applications, 6, 39-56.

https://doi.org/10.4236/csta.2017.63004

Received: August 1, 2017

Accepted: August 28, 2017

Published: August 31, 2017

Copyright $\odot 2017$ by authors and Scientific Research Publishing Inc. This work is licensed under the Creative Commons Attribution International License (CC BY 4.0).

http://creativecommons.org/licenses/by/4.0/

\begin{abstract}
The title compound, [N-(2-pyridylmethyl)-(L)-alanine] $\mathrm{Co}$ (III) thiocyanate (1) was obtained from the reaction of $\mathrm{Co}\left(\mathrm{OOCH}_{3}\right)_{2} \cdot \mathrm{H}_{2} \mathrm{O}$ with the tridentate reduced Schiff base ligand, $\mathrm{N}$-(2-pyridylmethyl)-(L)-alanine (L) and $\mathrm{NH}_{4} \mathrm{SCN}$ and characterized by elemental analysis, IR, UV-visible, TGA and singlecrystal X-ray diffraction. Structural and spectroscopic analyses reveal $\left.\left[\mathrm{Co}(\mathrm{L})_{2}\right)\right] \mathrm{SCN}$ to be monomeric with Cobalt(III) adopting a pseudo-octahedral geometry, coordinating to two reduce Schiff base ligands. In the crystal lattice, the thiocyanate anion forms an intermolecular $\mathrm{SCN} . . \mathrm{HN}_{\text {amine }}$ hydrogen bond, while adjacent monomers are linked by intermolecular $\mathrm{O}_{\text {carboxyl }} \cdots \mathrm{HN}_{\text {amine }} \cdots \mathrm{H}$-bonds to form a supramolecular network. This work is therefore undertaken in an attempt to construct coordination framework structures of varying properties using the mixed-ligand strategy involving reduced Schiff bases and the thiocyanate ion.
\end{abstract}

\section{Keywords}

Cobalt(III), (L)-Alanine, Thiocyanate, Tridentate Reduced Schiff Base, Crystal Structure

\section{Introduction}

Cobalt complexes of Schiff base ligands are an important class of coordination compounds due to their structural diversity which display geometries ranging from tetrahedral, bipyramidal, square pyramidal to octahedral [1] [2] [3]. Co- 
balt(III) complexes are generally prepared by air oxidation of Co(II) ion in the presence of relatively oxidation-inert ligands, which exhibit relatively strong coordinating abilities [4] [5]. An effective method for the construction of coordination frameworks with interesting structures and properties is through mixedligand assemblies and reduced Schiff base ligands have been found to form flexible and multidentate network structures because of the reduction of the imine group, $(-\mathrm{C}=\mathrm{N}-)$ of the Schiff base, thus overcoming ligand instability in complexes [6] [7].

Reduced Schiff base ligands have been found to be relatively inert towards air oxidation and are strongly coordinating due to the reduction of the $\mathrm{N}=\mathrm{C}$ bond, giving rise to flexible multidentate ligands [8] [9]. Pseudohalides possess versatile bonding modes, which result in the formation of complexes with various dimensionalities. The thiocyanate ions have therefore been found to possess versatile coordination abilities which result in the formation of metal complexes of varied structures. Thiocyanate-containing metal complexes have attracted much attention due to their versatile binding modes and its propensity to coordinate using either the nitrogen or/and the sulphur donor-atom, thus affording a number of homo- and hetero-metallic discrete structural assemblies with specific structural features and properties [10].

Recent attention has been focused on the study of the coordination compounds of cobalt due to its varied oxidation states and their interesting structural, magnetic, electronic and optoelectronic properties [11]. Our focus has been on the synthesis of mixed ligand complexes involving tridentate reduced Schiff bases and the thiocyanate ion. The crystal structure of one such compound is reported here.

\section{Experimental}

\subsection{Physical Measurements}

Elemental analysis for carbon, nitrogen and hydrogen were carried out on a FLASH 2000 Organic Elemental Analyzer, CHNS-O analyser by Thermo Scientific. Thermo Scientific iCAP 6000 SERIES duo ICAP Spectrometer was used to determine the metal content. Thermogravimetric analysis was investigated using a Mettler Toledo TGA/DSC1 STAR System; Infrared spectra were recorded on a Perkin-Elmer model IR-457 spectrometer and a spectrum 100 FT-IR Perkin Perkin-Elmer spectrometer, while the UV/Vis spectrum was recorded using an Agilent HP8453 Diode Array UV/Vis Spectrometer. The magnetic susceptibility measurement was made using the Sherwood Scientific magnetic susceptibility balance; while X-ray diffraction was carried using a Bruker APEX diffractometer.

\subsection{Synthesis and Crystallization}

$\mathrm{N}$-(2-pyridylmethyl)-(L)-alanine) (L)(0.361 $\mathrm{g}, 2 \mathrm{mmol})$ in $10 \mathrm{~mL}$ aqueous ethanol was added drop wise to a $5 \mathrm{ml}$ aqueous solution of $\mathrm{Co}\left(\mathrm{OOCH}_{3}\right)_{2} \cdot \mathrm{H}_{2} \mathrm{O}(0.249 \mathrm{~g}, 1$ $\mathrm{mmol}$,) while stirring at room temperature. After stirring for a further 10 minutes, 


\section{$\mathrm{Co}\left(\mathrm{OOCH}_{3}\right)_{2} \cdot \mathrm{H}_{2} \mathrm{O}+2 \mathrm{~L}$}



Scheme 1. Synthesis and structure of N-(2-pyridylmethyl)-L-alanine) isothiocyanate Cobalt(III).

$\mathrm{NH}_{4} \mathrm{SCN},(0.16 \mathrm{~g}, 2 \mathrm{mmol}$ ) $)$ in $2 \mathrm{~mL}$ distilled water was added drop wise and stirring continued for a further two hours. Rectangular reddish brown crystals suitable for X-ray analysis were obtained from the solution by slow evaporation. Yield: 81\%; anal. Calc. (Found) for $\mathrm{C}_{19} \mathrm{H}_{22} \mathrm{~N}_{5} \mathrm{O}_{4} \mathrm{SCo}$; C:48.00 (47.92); H:4.66 (4.78); N: 14.73 (14.72); S: 6.74 (6.69); Co: 12.40 (12.28). The synthesis of $\mathrm{N}$-(2-pyridylmethyl)-L-alanine) isothiocyanate Cobalt(III) is summarized in Scheme 1.

\subsection{Crystal Structure Determination}

A suitable single crystal of the title compound was mounted on a glass fiber on the goniometer head of a Bruker APEX diffractometer and data were collected using graphite monochromated $\mathrm{Cu}-K_{\alpha}$ radiation $(\lambda=1.54178 \AA$, operating at 50 $\mathrm{kV}$ and $40 \mathrm{~mA}$ ) at a temperature of $100 \mathrm{~K}$. Crystal data, data collection and structure refinement details are summarized in Table 1.

The structure was solved by direct methods and refined by full-matrix least squares on $F^{2}$ [12]. All non-Hydrogen atoms were refined anisotropically. $\mathrm{Hy}$ drogen atoms were included in calculated positions, assigned isotropic thermal parameters and allowed to ride on their parent carbon atoms. All calculations were carried out using the SHELXTL package [13]. CCDC 1419814 contains the supplementary crystallographic data for this paper.

\section{Results and Discussion}

\subsection{Spectroscopic and Other Analysis}

In the IR spectrum of the Co(III) complex, a very strong infrared absorption band at $1609 \mathrm{~cm}^{-1}$ is attributed to $v_{\mathrm{C}=\mathrm{C}}$ and $v_{\mathrm{C}=\mathrm{N}}$ of the pyridyl ring, while the strong band at $2098 \mathrm{~cm}^{-1}$ suggest the presence of $\mathrm{N}=\mathrm{C}=\mathrm{S}$ stretch [14] [15] [16], as confirmed by the single crystal structure of the compound. Two distinct peaks at $362 \mathrm{~nm}$ and $497 \mathrm{~nm}$ are observed in the UV-Visible spectrum of the complex and attributed to spin allowed $d$ - $d$ transitions.

The thermal behaviour of the title compound (Figure 1), recorded under an $\mathrm{N}_{2}$ atmosphere, in the temperature range of $25^{\circ} \mathrm{C}$ to $600^{\circ} \mathrm{C}$ at a heating rate of $25^{\circ} \mathrm{C} \mathrm{min}^{-1}$ shows a one-step decomposition patterns at $310^{\circ} \mathrm{C}$ though with a small shoulder at $240^{\circ} \mathrm{C}$ which probably accounts for the decomposition of the 
Table 1. Experimental details.

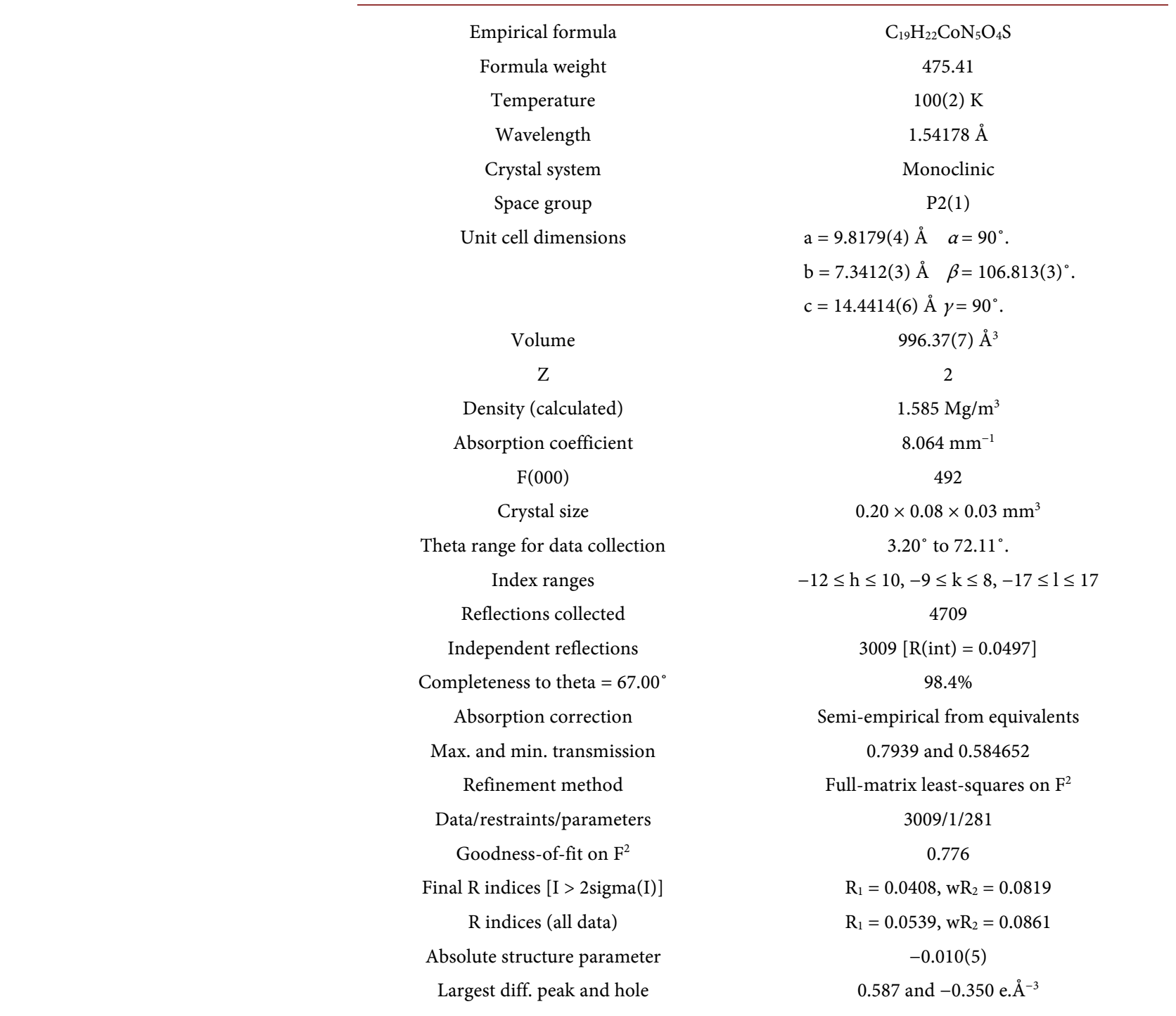



Figure 1. Thermal Analysis of the Title compound.

organic ligands [calc. $75.2 \%$ (found $75.8 \%$ )]. The final residue of [24.15\% (calc. 24.61\%)) represents a mixture of Cobalt oxides, $\mathrm{CoO}$ and $\mathrm{Co}_{2} \mathrm{O}_{3}$.

The peak at $\mathrm{m} / \mathrm{z}=474.1$ corresponds to $\mathrm{C}_{19} \mathrm{H}_{22} \mathrm{~N}_{5} \mathrm{O}_{4} \mathrm{SCo}$ (calc. 475.41) which confirms the molecular weight of the compound. The effective magnetic moment, $\mu_{\text {eff }}$ was found to be consistent with a $\mathrm{d}^{6}$ low-spin configuration with some 
degree of spin-orbit coupling.

\subsection{Crystal Structure Determination}

The structure of the title compound (I) is represented in Scheme 1 and comprise the $\left[\mathrm{Co}(\mathrm{L})_{2}\right]^{+}$cation and the $\mathrm{NCS}^{-}$anion with the resulting complex having the composition $\left[\mathrm{Co}(\mathrm{L})_{2}\right]^{+} \mathrm{NCS}^{-}$. The two L ligands are bonded to the cobalt centre via their two pyridyl nitrogen atoms $(\mathrm{Co}(1)-\mathrm{N}(1), 1.931(3) \AA ; \quad \mathrm{Co}(1)-\mathrm{N}(3)$, $1.929(3) \AA)$, two secondary amine nitrogen atoms (Co(1)-N(2), 1.971(4) $\mathrm{Co}(1)-\mathrm{N}(4), 1.967(3) \AA)$ and two carboxylate oxygen atoms $(\mathrm{Co}(1)-\mathrm{O}(2)$, 1.884(3) $\AA$; $\mathrm{Co}(1)-\mathrm{O}(4), 1.891(3) \AA$ ) to give a pseudo octahedral geometry around the cobalt atom (Figure 2 and Table 2). The X-ray crystal structure reveals that the thiocyanate anion in the crystal lattice is not bonded to the Co(III) centre but forms interionic hydrogen bonds [17] [18], through the nitrogen atom of the $\mathrm{NCS}^{-}$anion, [N(4)-H(4A)...N(5)\#1 (Figure 3, Table 3)]. A second intermolecular hydrogen bond, $[\mathrm{N}(2)-\mathrm{H}(2 \mathrm{~A}) \ldots \mathrm{O}(3) \# 2$ (Table 3)] is observed linking two different monomeric centres. The crystallographic indicators show a good-quality data set $[\mathrm{R}(\mathrm{int})=0.0497$ and $\mathrm{R} 1$ (all data) $=0.0539]$. Comparable bonds lengths and bond angles are observed. The average Co- $\mathrm{N}$ bond length $(1.950 \AA)$ is longer than the average Co-O bond length (1.886 $\AA$ ) as expected, and these values are in good agreement with those of similar cobalt(III) complexes [19]. The trans bond angles around the cobalt center, [N(3)-Co(1)- $\mathrm{N}(1)$ $\left.\left(174.18(16)^{\circ}\right), \mathrm{O}(2)-\mathrm{Co}(1)-\mathrm{N}(4)\left(171.62(16)^{\circ}\right), \mathrm{O}(4)-\mathrm{Co}(1)-\mathrm{N}(2)\left(172.66(16)^{\circ}\right)\right]$ deviates slightly from the theoretical value of $180^{\circ}$, some of the cis bond angles deviated considerably from the theoretical value of $90^{\circ}[\mathrm{N}(1)-\mathrm{Co}(1)-\mathrm{N}(2)$ bond angle of $94.30(18)^{\circ}$ [19]]. The bond angle of the N-C-S thiocyanate anion is within the expected range of $178^{\circ}-182^{\circ}$ [18]. The packing diagramme of the title compound is shown in Figure 4.

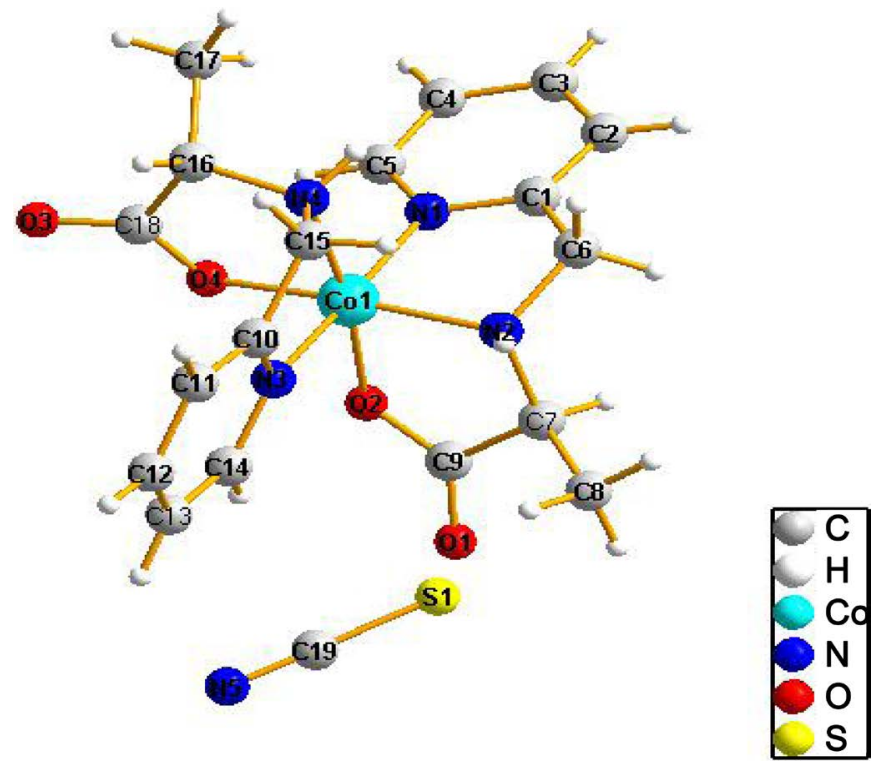

Figure 2. Ortep drawing of the Monomer of the titled compound (I) showing the atom numbering scheme. 
Table 2. Selected bond parameters $\left(\AA{ }^{\circ}\right)$.

\begin{tabular}{cc}
\hline $\mathrm{Co}(1)-\mathrm{O}(2)$ & $1.884(3)$ \\
$\mathrm{Co}(1)-\mathrm{O}(4)$ & $1.891(3)$ \\
$\mathrm{Co}(1)-\mathrm{N}(3)$ & $1.929(3)$ \\
$\mathrm{Co}(1)-\mathrm{N}(1)$ & $1.931(3)$ \\
$\mathrm{Co}(1)-\mathrm{N}(4)$ & $1.967(3)$ \\
$\mathrm{Co}(1)-\mathrm{N}(2)$ & $1.971(4)$ \\
$\mathrm{O}(3)-\mathrm{C}(18)-\mathrm{O}(4)$ & $122.9(4)$ \\
$\mathrm{N}(5)-\mathrm{C}(19)-\mathrm{S}(1)$ & $178.9(4)$ \\
$\mathrm{O}(2)-\mathrm{Co}(1)-\mathrm{O}(4)$ & $86.73(14)$ \\
$\mathrm{O}(2)-\mathrm{Co}(1)-\mathrm{N}(3)$ & $91.93(13)$ \\
$\mathrm{O}(4)-\mathrm{Co}(1)-\mathrm{N}(3)$ & $88.55(17)$ \\
$\mathrm{O}(2)-\mathrm{Co}(1)-\mathrm{N}(1)$ & $93.53(13)$ \\
$\mathrm{O}(4)-\mathrm{Co}(1)-\mathrm{N}(1)$ & $93.77(18)$ \\
$\mathrm{N}(3)-\mathrm{Co}(1)-\mathrm{N}(1)$ & $174.18(16)$ \\
$\mathrm{O}(2)-\mathrm{Co}(1)-\mathrm{N}(4)$ & $171.62(16)$ \\
$\mathrm{O}(4)-\mathrm{Co}(1)-\mathrm{N}(4)$ & $86.88(17)$ \\
$\mathrm{N}(3)-\mathrm{Co}(1)-\mathrm{N}(4)$ & $82.50(14)$ \\
$\mathrm{N}(1)-\mathrm{Co}(1)-\mathrm{N}(4)$ & $92.29(14)$ \\
$\mathrm{O}(2)-\mathrm{Co}(1)-\mathrm{N}(2)$ & $86.41(15)$ \\
$\mathrm{O}(4)-\mathrm{Co}(1)-\mathrm{N}(2)$ & $172.66(16)$ \\
$\mathrm{N}(3)-\mathrm{Co}(1)-\mathrm{N}(2)$ & $94.30(18)$ \\
$\mathrm{N}(1)-\mathrm{Co}(1)-\mathrm{N}(2)$ & $84.03(18)$ \\
$\mathrm{N}(4)-\mathrm{Co}(1)-\mathrm{N}(2)$ & \\
\hline & \\
\hline
\end{tabular}

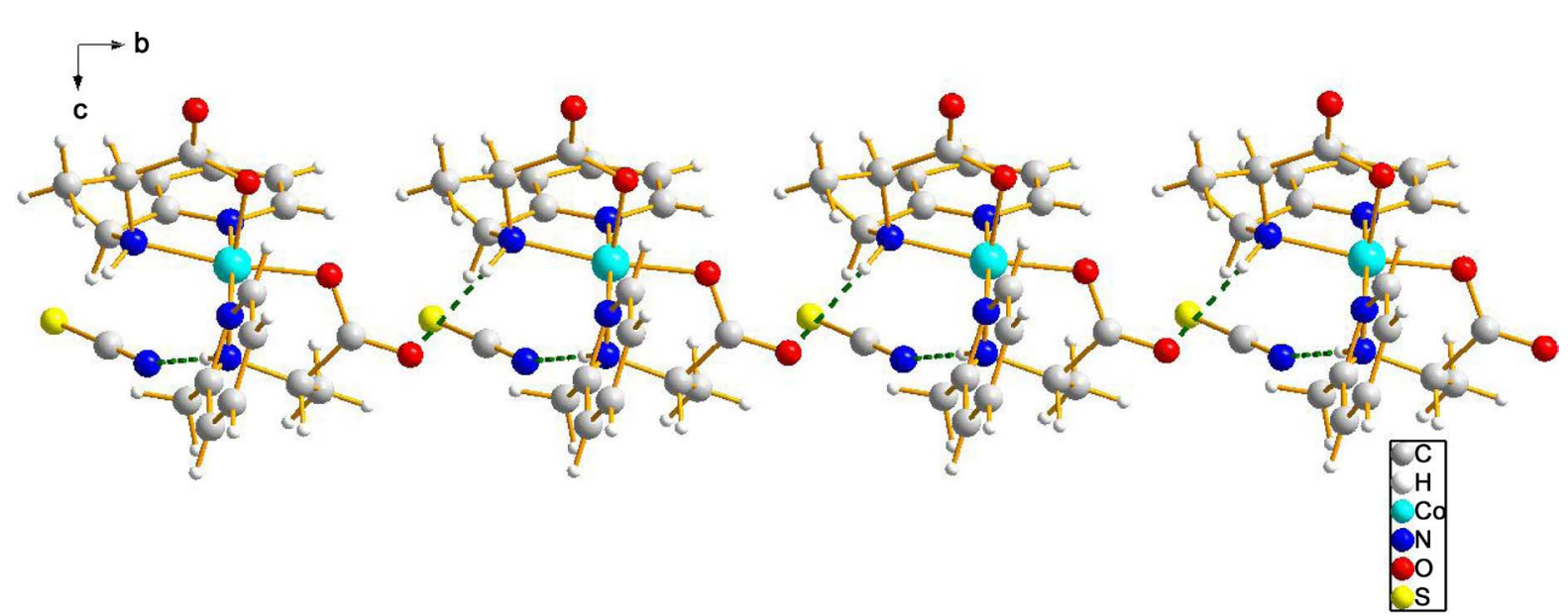

Figure 3. Ortep diagram showing hydrogen bonding.

Table 3. Hydrogen bonds ( $\AA$ and ${ }^{\circ}$ ].

\begin{tabular}{ccccc}
\hline $\mathrm{D}-\mathrm{H} \ldots \mathrm{A}$ & $\mathrm{d}(\mathrm{D}-\mathrm{H})$ & $\mathrm{d}(\mathrm{H} \ldots \mathrm{A})$ & $\mathrm{d}(\mathrm{D} \ldots \mathrm{A})$ & $<(\mathrm{DHA})$ \\
\hline $\mathrm{N}(4)-\mathrm{H}(4 \mathrm{~A}) \ldots \mathrm{N}(5) \# 1$ & $0.87(4)$ & $2.03(5)$ & $2.892(5)$ & $174(4)$ \\
$\mathrm{N}(2)-\mathrm{H}(2 \mathrm{~A}) \ldots \mathrm{O}(3) \# 2$ & $0.88(5)$ & $2.26(5)$ & $2.997(5)$ & $141(4)$
\end{tabular}

Symmetry transformations used to generate equivalent atoms: $\# 1 \mathrm{x}-1, \mathrm{y}, \mathrm{z} \# 2 \mathrm{x}, \mathrm{y}-1, \mathrm{z}$. 


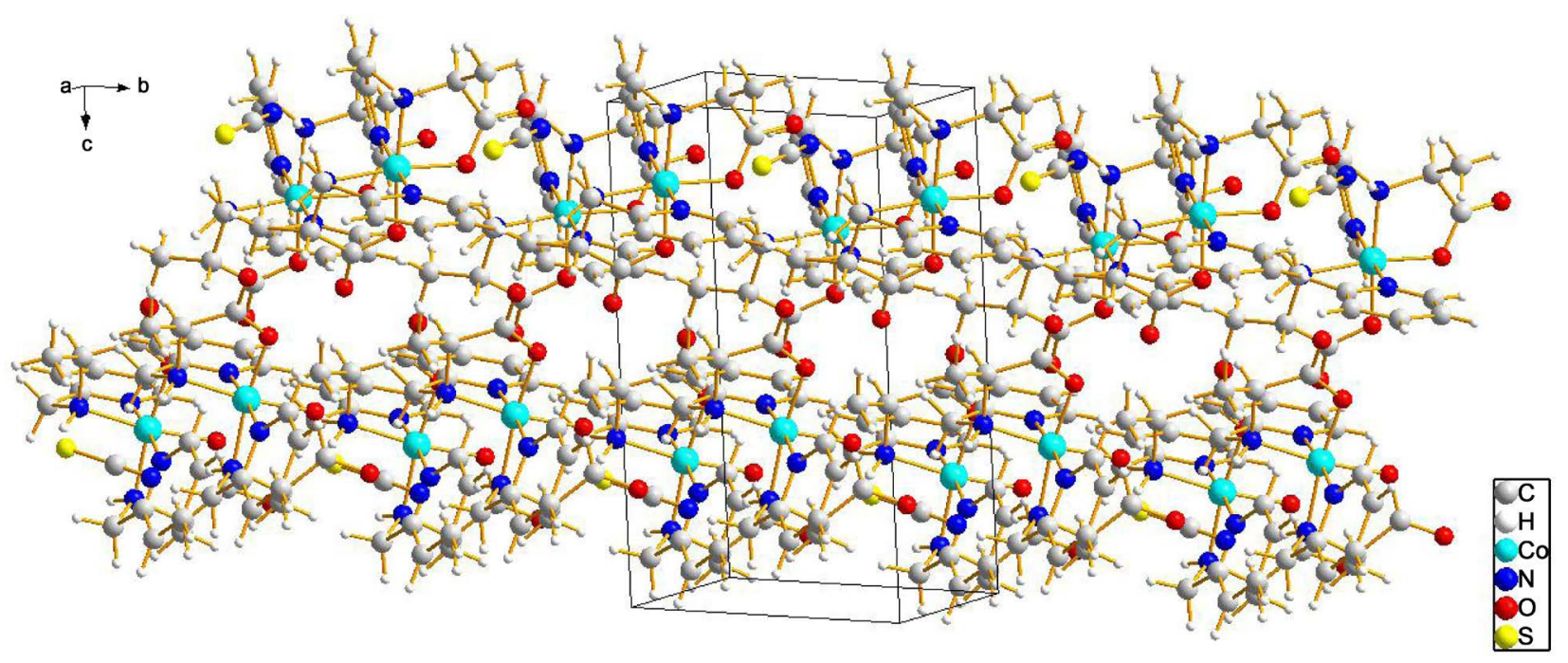

Figure 4. Packing diagram the title compound.

\section{Conclusion}

We have isolated a Co(III) Isothiocyanate metal-organic framework containing the chiral ligand, N-(2-pyridylmethyl)-L-alanine (pyala). Spectroscopic studies and the $\mathrm{x}$-ray structure show that the compound is a monomer in which Cobalt(III) adopts a pseudo octahedral geometry, coordinating to two molecules of the ligand. Adjacent monomeric centres are linked by N-H...O, N-H...S hydrogen bonds which stabilize the compound thus forming a $3 \mathrm{D}$ supramolecular networks.

\section{Acknowledgements}

The authors thank the Royal Society-DFID capacity building initiative program for mobility bursary to (NS-JE).

\section{Conflict of Interest}

The authors declare that there is no conflict of interest.

\section{References}

[1] Vafazadeh, R. and Kashfi, M. (2007) Synthesis and Characterization of Cobalt(III) Octahedral Complexes with Flexible Salpn Schiff Base in Solution. Structural Dependence of the Complexes on the Nature of Schiff Base and Axial Ligands. Bulletin of the Korean Chemical Society, 28, 1227-1230. https://doi.org/10.5012/bkcs.2007.28.7.1227

[2] Meghdadi, S., Amirnasr, M., Mereiter, K., Molaee, H. and Amiri, A. (2011) Synthesis, Structure and Electrochemistry of Co(III) Complexes with an Unsymmetrical Schiff Base Ligand Derived from 2-Aminobenzylamine and Pyrrole-2-Carboxaldehyde. Polyhedron, 30, 1651-1656. https://doi.org/10.1016/j.poly.2011.03.041

[3] Perkins, C.M., Rose, N.J. and Stenkamp, R.E. (1990) The Structure of Cobalt Methylmalonate Complexes, $\mathrm{CoClN}_{4} \mathrm{O}_{7} \mathrm{C}_{10} \mathrm{H}_{28}$ and $\mathrm{CoCl}_{2} \mathrm{~N}_{4} \mathrm{O}_{5.5} \mathrm{C}_{11} \mathrm{H}_{28}$, Models for Metal Complexes of $\gamma$-Carboxyglutamic Acid. Inorganica Chimica Acta, 172, 119-125. 
https://doi.org/10.1016/S0020-1693(00)80460-0

[4] Costa, G., Mestroni, G. and Cocevar, C. (1971) Cobalt Chelates as Models for Methyl Donors and Acceptors in Vitamin $\mathrm{B}_{12}$-Dependent Transmethylation. Direct Methylation of Vitamin $\mathrm{B}_{12 \mathrm{a}}$. Journal of the Chemical Society D, 13, 706. https://doi.org/10.1039/c2971000706b

[5] Martín, C., Whiteoak, C.J., Martin, E., Escudero-Adán, E.C., Galán-Mascarós, J.R. and Kleij, A.W. (2014) Synthesis and Structural Features of Co(II) and Co(III) Complexes Supported by Aminotrisphenolate Ligand Scaffolds. Inorganic Chemistry, 53, 11675-11681. https://doi.org/10.1021/ic501883z

[6] Zheng, Y., Zhou, Y.-L., Zeng, M.-H. and Kurmoo, M. (2015) The Concept of Mixed-Organic Frameworks: Design, Turnig and Functions. Dalton Transactions, 44, 5258-5275. https://doi.org/10.1039/C4DT04030A

[7] Yang, C.T., Moubaraki, B., Murray, K.S., Ranford, J.D. and Vittal, J.J. (2001) Interconversion of a Monomer and Two Coordination Polymers of a Copper(II)-Reduced Schiff Base Ligand-1, 10-Phenanthroline Complex Based on Hydrogen-and Coordinative-Bonding. Inorganic Chemistry, 40, 5934-5941.

https://doi.org/10.1021/ic010479b

[8] Ganguly, R., Sreenivasulu, B. and Vittal, J.J. (2008) Amino Acid-Containing Reduced Schiff Bases as the Building Blocks for Metallasupramolecular Structures. Coordination Chemistry Reviews, 252, 1027-1050.

https://doi.org/10.1016/j.ccr.2008.01.005

[9] Sreenivasulu, B. and Vittal, J.J. (2009) Hydrogen-Bonded Copper (II) and Nickel (II) Complexes and Coordination Polymeric Structures Containing Reduced Schiff Base Ligands. Inorganica Chimica Acta, 362, 2735-2743. https://doi.org/10.1016/j.ica.2008.12.018

[10] Buckingham, D.A. (1994) The Linkage Isomerism of Thiocyanate Bonded to Cobalt (III). Coordination Chemistry Reviews, 135, 587-621. https://doi.org/10.1016/0010-8545(94)80078-2

[11] Ndifon, P.T., Agwara, M.O., Paboudam, A.G., Yufanyi, D.M., Ngoune, J., Galindo, A., Álvarez, E. and Mohamadou, A. (2009) Synthesis, Characterisation and Crystal Structure of a Cobalt(II)-Hexamethylenetetramine Coordination Polymer. Transition Metal Chemistry, 34, 745-750. https://doi.org/10.1007/s11243-009-9257-1

[12] Sheldrick, G.M. (1997) SHELXL97, Program for Crystal Structure Refinement. University of Gottingen, Gottingen.

[13] Sheldrick, G.M. (2001) SHELXTL, Version 6.1. Bruker AXS, Inc., Maddison, WI.

[14] Malecki, G., Gron, T. and Duda. H. (2012) Structural, Spectroscopic and Magnetic Properties of Thiocyanate Complexes of $\mathrm{Mn}(\mathrm{II}), \mathrm{Ni}(\mathrm{II})$ and $\mathrm{Cu}(\mathrm{II})$ with the 1-Methylimidazole Ligand. Polyhedron, 36, 56-68.

https://doi.org/10.1016/j.poly.2012.01.026

[15] Chavez, F.A. and Mascharak, P.K. (2000) Co (III)-Alkylperoxo Complexes: Syntheses, Structure-Reactivity Correlations, and Use in the Oxidation of Hydrocarbons. Accounts of Chemical Research, 33, 539-545. https://doi.org/10.1021/ar990089h

[16] Murugavel, R., Choudhury, A., Walawalkar, M.G., Pothiraja, R. and Rao, C.N.R. (2008) Metal Complexes of Organophosphate Esters and Open-Framework Metal Phosphates: Synthesis, Structure, Transformations, and Applications. Chemical Reviews, 108, 3549-3655. https://doi.org/10.1021/cr000119q

[17] Khan, S., Bhar, K., Roy, S., Mitra, P., Slawin, A.M.Z. and Ghosh, B.K. (2011) Synthesis, Structures and Molecular Properties of Two Mononuclear Cobalt(III) Halide 
Complexes Containing a Tetradentate N-Donor Schiff Base as End-Capping Ligand. Indian Journal of Chemistry, 50A, 1429-1435.

[18] Pariya, C., Chaudhuri, N.R., Das, A. and Seth, S.K. (1995) Crystal Structure of Bis (N1-Isopropyl-2-Methyl-1,2-Propanediamine)Isothiocyanatozinc (II) Thiocyanate. Acta Chemica Scandinavica, 49, 859-864. https://doi.org/10.3891/acta.chem.scand.49-0859

[19] Layek, M., Ghosh, M., Sain, S., Fleck, M., Muthiah, P.T., Jenniefer, S.J., Ribas, J. and Bandyopadhyay, D. (2013) Synthesis, Crystal Structure and Magnetic Properties of Nickel (II) and Cobalt (III) Complexes of a Pentadentate Schiff Base. Journal of Molecular Structure, 1036, 422-426. https://doi.org/10.1016/j.molstruc.2012.11.068 


\section{Supplementary Materials}

\section{[N-(2-pyridylmethyI) -(L)-alanine] Co(III) thiocyanate \\ Computing details}

Data collection: Bruker Apex diffractometer; Data collection: Graphite monochromated $\mathrm{Cu}-\mathrm{k} \alpha$ radiation $(\lambda=1.54178 \AA$ ); structure solved by: Direct methods; refined by: Full matrix least square method; all calculations were carried out by: SHELXTL package.

Crystal data

\begin{tabular}{cc}
\hline $\mathrm{C}_{19} \mathrm{H}_{22} \mathrm{~N}_{5} \mathrm{O}_{4} \mathrm{SCo}$ & $\mathrm{F}(000)=492$ \\
$M r=475.41$ & $D X=1.585 \mathrm{Mg} / \mathrm{m}^{3}$ \\
Monoclinic, $\mathrm{P} 2(1)$ & MoKa radiation, $\lambda=1.54178 \AA$ \\
$\mathrm{a}=9.8179(4) \AA$ & Cell parameters from 4709 reflections \\
$\mathrm{b}=7.3412(3) \AA$ & $\theta=3.20$ to $72.11^{\circ}$. \\
$\mathrm{c}=14.4414(6) \AA$ & $\mu=8.064 \mathrm{~mm}^{-1}$ \\
$V=996.37(7) \AA^{3}$ & $T=100(2) \mathrm{K}$ \\
$Z=2$ & $0.20 \times 0.08 \times 0.03 \mathrm{~mm}^{3}$ \\
\hline
\end{tabular}

Data collection

Bruker APEX diffractometer

Radiation source: graphite monochromated $\mathrm{Cu}-K_{\alpha}$ radiation

Absorption correction: Semi-empirical from equivalents

$T_{\min }=0.584652, T_{\max }=0.7939$

4709 measured reflections
3009 Independent reflections

3009 reflections with $\mathrm{I}>2$ sigma(I)

$$
\mathrm{R} \text { (int) }=0.0497
$$

$\theta_{\max }=72.11^{\circ}, \theta_{\min }=3.20^{\circ}$

$h=-12 \rightarrow 10$

$k=-9 \rightarrow 8$

$I=-17 \rightarrow 17$

Refinement

\begin{tabular}{cc}
\hline Refinement on $\mathrm{F}^{2}$ & 3009 reflections \\
Full-matrix least-squares & 281 parameters \\
$\mathrm{R}_{1}=0.0539, \mathrm{wR}_{2}=0.0861$ & 1 restrains \\
$S=-0.010(5)$ & \\
\hline
\end{tabular}

Table S1. Atomic coordinates $(\times 104)$ and equivalent isotropic displacement parameters $(\AA 2 \times 103) . \mathrm{U}(\mathrm{eq})$ is defined as one third of the trace of the orthogonalized Uij tensor.

\begin{tabular}{ccccc}
\hline & $\mathrm{x}$ & $\mathrm{y}$ & $\mathrm{z}$ & $\mathrm{U}(\mathrm{eq})$ \\
\hline $\mathrm{C}(1)$ & $1690(5)$ & $4175(7)$ & $3505(3)$ & $15(1)$ \\
$\mathrm{C}(2)$ & $583(5)$ & $3899(7)$ & $3909(3)$ & $21(1)$ \\
$\mathrm{C}(3)$ & $-123(5)$ & $5428(7)$ & $4109(3)$ & $22(1)$ \\
$\mathrm{C}(4)$ & $331(5)$ & $7165(8)$ & $3942(3)$ & $22(1)$ \\
$\mathrm{C}(5)$ & $1451(5)$ & $7319(7)$ & $3555(3)$ & $19(1)$ \\
\hline
\end{tabular}




\section{Continued}

\begin{tabular}{lllll}
\hline $\mathrm{C}(6)$ & $2428(4)$ & $2659(6)$ & $3169(3)$ & $14(1)$ \\
$\mathrm{C}(7)$ & $5010(5)$ & $3078(7)$ & $3978(3)$ & $17(1)$ \\
$\mathrm{C}(8)$ & $6004(5)$ & $1571(6)$ & $3869(3)$ & $23(1)$ \\
$\mathrm{C}(9)$ & $5752(5)$ & $4892(7)$ & $4298(3)$ & $18(1)$ \\
$\mathrm{C}(10)$ & $4486(4)$ & $5285(6)$ & $1065(3)$ & $16(1)$ \\
$\mathrm{C}(11)$ & $5308(5)$ & $5304(6)$ & $431(3)$ & $19(1)$ \\
$\mathrm{C}(12)$ & $6703(4)$ & $5895(8)$ & $772(3)$ & $19(1)$ \\
$\mathrm{C}(13)$ & $7235(5)$ & $6450(6)$ & $1727(3)$ & $20(1)$ \\
$\mathrm{C}(14)$ & $6361(4)$ & $6430(6)$ & $2313(3)$ & $18(1)$ \\
$\mathrm{C}(15)$ & $2951(4)$ & $4702(7)$ & $800(3)$ & $16(1)$ \\
$\mathrm{C}(16)$ & $1945(5)$ & $7696(6)$ & $1036(3)$ & $18(1)$ \\
$\mathrm{C}(17)$ & $404(5)$ & $8204(7)$ & $948(4)$ & $21(1)$ \\
$\mathrm{C}(18)$ & $2918(5)$ & $9048(6)$ & $1701(3)$ & $16(1)$ \\
$\mathrm{C}(19)$ & $9040(5)$ & $2628(7)$ & $1589(3)$ & $20(1)$ \\
$\mathrm{Co}(1)$ & $3622(1)$ & $5922(1)$ & $2717(1)$ & $12(1)$ \\
$\mathrm{N}(1)$ & $2112(3)$ & $5869(7)$ & $3327(2)$ & $15(1)$ \\
$\mathrm{N}(2)$ & $3808(4)$ & $3315(5)$ & $3055(3)$ & $14(1)$ \\
$\mathrm{N}(3)$ & $5000(3)$ & $5844(6)$ & $2000(2)$ & $15(1)$ \\
$\mathrm{N}(4)$ & $2268(3)$ & $5787(6)$ & $1414(2)$ & $14(1)$ \\
$\mathrm{N}(5)$ & $9758(4)$ & $3657(6)$ & $1330(3)$ & $23(1)$ \\
$\mathrm{O}(1)$ & $6903(3)$ & $4966(5)$ & $4911(2)$ & $20(1)$ \\
$\mathrm{O}(2)$ & $5048(3)$ & $6324(4)$ & $3892(2)$ & $14(1)$ \\
$\mathrm{O}(3)$ & $3036(3)$ & $84609(5)$ & $1433(2)$ & $21(1)$ \\
$\mathrm{O}(4)$ & $3549(3)$ & $2563(2)$ & $15(1)$ \\
$\mathrm{S}(1)$ & $8003(1)$ & $1938(1)$ & \\
\hline & & & & \\
& & & & \\
\hline
\end{tabular}

Table S2. Hydrogen coordinates $(\times 104)$ and isotropic displacement parameters $(\AA 2 \times$ 103).

\begin{tabular}{ccccc}
\hline & $x$ & $y$ & $z$ & U(eq) \\
\hline$H(2)$ & 317 & 2706 & 4045 & 25 \\
$\mathrm{H}(3)$ & -912 & 5289 & 4359 & 26 \\
$\mathrm{H}(4)$ & -124 & 8219 & 4093 & 26 \\
$\mathrm{H}(5)$ & 1766 & 8501 & 3445 & 23 \\
$\mathrm{H}(6 \mathrm{~A})$ & 1821 & 2191 & 2543 & 17 \\
$\mathrm{H}(6 \mathrm{~B})$ & 2603 & 1651 & 3645 & 17 \\
$\mathrm{H}(7)$ & 4574 & 2689 & 4493 & 21 \\
$\mathrm{H}(8 \mathrm{~A})$ & 5467 & 433 & 3699 & 35 \\
$\mathrm{H}(8 \mathrm{~B})$ & 6754 & 1410 & 4481 & 35 \\
$\mathrm{H}(8 \mathrm{C})$ & 6434 & 1892 & 3358 & 35 \\
$\mathrm{H}(11)$ & 4924 & 4923 & -221 & 23 \\
$\mathrm{H}(12)$ & 7294 & 5920 & 354 & 23 \\
$\mathrm{H}(13)$ & 8195 & 6841 & 1972 & 24 \\
$\mathrm{H}(14)$ & 6723 & 6841 & 2962 & 22
\end{tabular}


Continued

\begin{tabular}{ccccc}
\hline $\mathrm{H}(15 \mathrm{~A})$ & 2479 & 4941 & 106 & 20 \\
$\mathrm{H}(15 \mathrm{~B})$ & 2881 & 3384 & 923 & 20 \\
$\mathrm{H}(16)$ & 2097 & 7774 & 381 & 21 \\
$\mathrm{H}(17 \mathrm{~A})$ & -238 & 7380 & 489 & 32 \\
$\mathrm{H}(17 \mathrm{~B})$ & 225 & 9460 & 716 & 32 \\
$\mathrm{H}(17 \mathrm{C})$ & 236 & 8097 & 1582 & 32 \\
$\mathrm{H}(4 \mathrm{~A})$ & $1490(50)$ & $5220(60)$ & $1400(30)$ & $7(11)$ \\
$\mathrm{H}(2 \mathrm{~A})$ & $4030(50)$ & $2630(80)$ & $2620(40)$ & $20(15)$ \\
\hline
\end{tabular}

Table S3. Anisotropic displacement parameters $(\AA 2 \times 103)$. The anisotropic displacement factor exponent takes the form: $-2 \mathrm{p} 2\left[\mathrm{~h} 2 \mathrm{a} * 2 \mathrm{U} 11+\ldots+2 \mathrm{hka}{ }^{\star} \mathrm{b}{ }^{\star} \mathrm{U} 12\right]$.

\begin{tabular}{|c|c|c|c|c|c|c|}
\hline & U11 & U22 & U33 & $\mathrm{U} 23$ & U13 & U12 \\
\hline $\mathrm{C}(1)$ & $17(2)$ & $15(3)$ & $11(2)$ & $2(2)$ & $2(2)$ & $0(2)$ \\
\hline$C(2)$ & $23(2)$ & $17(3)$ & $21(2)$ & $5(2)$ & $5(2)$ & $1(2)$ \\
\hline$C(3)$ & $19(2)$ & $30(3)$ & $16(2)$ & $-4(2)$ & $5(2)$ & $-4(2)$ \\
\hline $\mathrm{C}(4)$ & $25(2)$ & $22(3)$ & $17(2)$ & $-6(2)$ & $5(2)$ & $3(2)$ \\
\hline$C(5)$ & $21(2)$ & $12(2)$ & $21(2)$ & $-3(2)$ & $2(2)$ & $-2(2)$ \\
\hline$C(6)$ & $16(2)$ & $10(2)$ & $18(2)$ & $2(2)$ & $7(2)$ & $3(2)$ \\
\hline $\mathrm{C}(7)$ & $17(2)$ & $15(3)$ & $18(2)$ & $6(2)$ & $3(2)$ & $0(2)$ \\
\hline $\mathrm{C}(8)$ & $21(2)$ & $14(3)$ & $29(2)$ & $-6(2)$ & $0(2)$ & $5(2)$ \\
\hline $\mathrm{C}(9)$ & $21(2)$ & $22(3)$ & $15(2)$ & $-2(2)$ & $8(2)$ & $3(2)$ \\
\hline$C(10)$ & $17(2)$ & $9(2)$ & $22(2)$ & $2(2)$ & $5(2)$ & $0(2)$ \\
\hline$C(11)$ & $23(2)$ & $13(2)$ & $22(2)$ & $2(2)$ & $6(2)$ & $4(2)$ \\
\hline$C(12)$ & $22(2)$ & $16(2)$ & $24(2)$ & $3(2)$ & $14(2)$ & $6(2)$ \\
\hline$C(13)$ & $15(2)$ & $20(3)$ & $25(2)$ & $1(2)$ & $5(2)$ & $0(2)$ \\
\hline$C(14)$ & $17(2)$ & $20(3)$ & $18(2)$ & $3(2)$ & $6(2)$ & $-1(2)$ \\
\hline$C(15)$ & $19(2)$ & $13(2)$ & $16(2)$ & $-4(2)$ & $3(2)$ & $-2(2)$ \\
\hline$C(16)$ & $21(2)$ & $13(2)$ & $19(2)$ & $3(2)$ & $7(2)$ & $2(2)$ \\
\hline$C(17)$ & $19(2)$ & $18(3)$ & $28(2)$ & $3(2)$ & $9(2)$ & $-1(2)$ \\
\hline$C(18)$ & $22(2)$ & $13(2)$ & $16(2)$ & $1(2)$ & $8(2)$ & $2(2)$ \\
\hline$C(19)$ & $15(2)$ & $26(3)$ & $17(2)$ & $-3(2)$ & $3(2)$ & $4(2)$ \\
\hline $\mathrm{Co}(1)$ & $14(1)$ & $10(1)$ & $13(1)$ & $0(1)$ & $4(1)$ & $0(1)$ \\
\hline $\mathrm{N}(1)$ & $14(2)$ & $16(2)$ & $17(2)$ & $0(2)$ & $6(1)$ & $-3(2)$ \\
\hline $\mathrm{N}(2)$ & $18(2)$ & $11(2)$ & $16(2)$ & $-1(2)$ & $6(2)$ & $-1(2)$ \\
\hline $\mathrm{N}(3)$ & $16(2)$ & $11(2)$ & $18(2)$ & $2(2)$ & $6(1)$ & $0(2)$ \\
\hline $\mathrm{N}(4)$ & $10(1)$ & $14(2)$ & $16(2)$ & $-2(2)$ & $4(1)$ & $-4(2)$ \\
\hline $\mathrm{N}(5)$ & $19(2)$ & $29(3)$ & $20(2)$ & $-2(2)$ & $6(2)$ & $-3(2)$ \\
\hline $\mathrm{O}(1)$ & $22(2)$ & $19(2)$ & $16(2)$ & $2(1)$ & $-2(1)$ & $1(1)$ \\
\hline $\mathrm{O}(2)$ & $18(1)$ & $12(2)$ & $12(1)$ & $0(1)$ & $4(1)$ & $-1(1)$ \\
\hline $\mathrm{O}(3)$ & $32(2)$ & $12(2)$ & $20(1)$ & $2(1)$ & $9(1)$ & $-1(1)$ \\
\hline $\mathrm{O}(4)$ & $19(2)$ & $10(2)$ & $16(2)$ & $0(1)$ & $6(1)$ & $1(1)$ \\
\hline $\mathrm{S}(1)$ & $35(1)$ & $24(1)$ & $32(1)$ & $-2(1)$ & $18(1)$ & $-8(1)$ \\
\hline
\end{tabular}


Table S4. Bond lengths $[\AA ̊]$ and angles [ $\left.{ }^{\circ}\right]$.

\begin{tabular}{|c|c|}
\hline $\mathrm{C}(1)-\mathrm{N}(1)$ & $1.359(6)$ \\
\hline $\mathrm{C}(1)-\mathrm{C}(2)$ & $1.388(7)$ \\
\hline$C(1)-C(6)$ & $1.484(6)$ \\
\hline $\mathrm{C}(2)-\mathrm{C}(3)$ & $1.393(7)$ \\
\hline $\mathrm{C}(2)-\mathrm{H}(2)$ & 0.9500 \\
\hline $\mathrm{C}(3)-\mathrm{C}(4)$ & $1.394(7)$ \\
\hline $\mathrm{C}(3)-\mathrm{H}(3)$ & 0.9500 \\
\hline$C(4)-C(5)$ & $1.375(6)$ \\
\hline $\mathrm{C}(4)-\mathrm{H}(4)$ & 0.9500 \\
\hline $\mathrm{C}(5)-\mathrm{N}(1)$ & $1.337(6)$ \\
\hline $\mathrm{C}(5)-\mathrm{H}(5)$ & 0.9500 \\
\hline $\mathrm{C}(6)-\mathrm{N}(2)$ & $1.491(5)$ \\
\hline $\mathrm{C}(6)-\mathrm{H}(6 \mathrm{~A})$ & 0.9900 \\
\hline $\mathrm{C}(6)-\mathrm{H}(6 \mathrm{~B})$ & 0.9900 \\
\hline $\mathrm{C}(7)-\mathrm{N}(2)$ & $1.513(5)$ \\
\hline $\mathrm{C}(7)-\mathrm{C}(8)$ & $1.513(6)$ \\
\hline $\mathrm{C}(7)-\mathrm{C}(9)$ & $1.524(6)$ \\
\hline $\mathrm{C}(7)-\mathrm{H}(7)$ & 1.0000 \\
\hline $\mathrm{C}(8)-\mathrm{H}(8 \mathrm{~A})$ & 0.9800 \\
\hline $\mathrm{C}(8)-\mathrm{H}(8 \mathrm{~B})$ & 0.9800 \\
\hline $\mathrm{C}(8)-\mathrm{H}(8 \mathrm{C})$ & 0.9800 \\
\hline $\mathrm{C}(9)-\mathrm{O}(1)$ & $1.218(5)$ \\
\hline $\mathrm{C}(9)-\mathrm{O}(2)$ & $1.301(6)$ \\
\hline $\mathrm{C}(10)-\mathrm{N}(3)$ & $1.361(5)$ \\
\hline$C(10)-C(11)$ & $1.384(6)$ \\
\hline$C(10)-C(15)$ & $1.505(6)$ \\
\hline$C(11)-C(12)$ & $1.385(6)$ \\
\hline $\mathrm{C}(11)-\mathrm{H}(11)$ & 0.9500 \\
\hline$C(12)-C(13)$ & $1.387(6)$ \\
\hline $\mathrm{C}(12)-\mathrm{H}(12)$ & 0.9500 \\
\hline $\mathrm{C}(13)-\mathrm{C}(14)$ & $1.369(6)$ \\
\hline $\mathrm{C}(13)-\mathrm{H}(13)$ & 0.9500 \\
\hline $\mathrm{C}(14)-\mathrm{N}(3)$ & $1.350(5)$ \\
\hline $\mathrm{C}(14)-\mathrm{H}(14)$ & 0.9500 \\
\hline $\mathrm{C}(15)-\mathrm{N}(4)$ & $1.489(6)$ \\
\hline $\mathrm{C}(15)-\mathrm{H}(15 \mathrm{~A})$ & 0.9900 \\
\hline $\mathrm{C}(15)-\mathrm{H}(15 \mathrm{~B})$ & 0.9900 \\
\hline $\mathrm{C}(16)-\mathrm{N}(4)$ & $1.505(6)$ \\
\hline$C(16)-C(18)$ & $1.514(6)$ \\
\hline$C(16)-C(17)$ & $1.527(6)$ \\
\hline $\mathrm{C}(16)-\mathrm{H}(16)$ & 1.0000 \\
\hline $\mathrm{C}(17)-\mathrm{H}(17 \mathrm{~A})$ & 0.9800 \\
\hline $\mathrm{C}(17)-\mathrm{H}(17 \mathrm{~B})$ & 0.9800 \\
\hline
\end{tabular}


Continued

\begin{tabular}{|c|c|}
\hline $\mathrm{C}(17)-\mathrm{H}(17 \mathrm{C})$ & 0.9800 \\
\hline $\mathrm{C}(18)-\mathrm{O}(3)$ & $1.226(5)$ \\
\hline $\mathrm{C}(18)-\mathrm{O}(4)$ & $1.288(5)$ \\
\hline $\mathrm{C}(19)-\mathrm{N}(5)$ & $1.166(6)$ \\
\hline$C(19)-S(1)$ & $1.643(5)$ \\
\hline $\mathrm{Co}(1)-\mathrm{O}(2)$ & $1.884(3)$ \\
\hline $\mathrm{Co}(1)-\mathrm{O}(4)$ & $1.891(3)$ \\
\hline $\mathrm{Co}(1)-\mathrm{N}(3)$ & $1.929(3)$ \\
\hline $\mathrm{Co}(1)-\mathrm{N}(1)$ & $1.931(3)$ \\
\hline $\mathrm{Co}(1)-\mathrm{N}(4)$ & $1.967(3)$ \\
\hline $\mathrm{Co}(1)-\mathrm{N}(2)$ & $1.971(4)$ \\
\hline $\mathrm{N}(2)-\mathrm{H}(2 \mathrm{~A})$ & $0.88(5)$ \\
\hline $\mathrm{N}(4)-\mathrm{H}(4 \mathrm{~A})$ & $0.87(4)$ \\
\hline $\mathrm{N}(1)-\mathrm{C}(1)-\mathrm{C}(2)$ & $122.1(4)$ \\
\hline $\mathrm{N}(1)-\mathrm{C}(1)-\mathrm{C}(6)$ & $114.8(4)$ \\
\hline$C(2)-C(1)-C(6)$ & $122.9(4)$ \\
\hline $\mathrm{C}(1)-\mathrm{C}(2)-\mathrm{C}(3)$ & $117.8(5)$ \\
\hline $\mathrm{C}(1)-\mathrm{C}(2)-\mathrm{H}(2)$ & 121.1 \\
\hline $\mathrm{C}(3)-\mathrm{C}(2)-\mathrm{H}(2)$ & 121.1 \\
\hline$C(2)-C(3)-C(4)$ & $119.8(4)$ \\
\hline $\mathrm{C}(2)-\mathrm{C}(3)-\mathrm{H}(3)$ & 120.1 \\
\hline $\mathrm{C}(4)-\mathrm{C}(3)-\mathrm{H}(3)$ & 120.1 \\
\hline$C(5)-C(4)-C(3)$ & $118.6(5)$ \\
\hline $\mathrm{C}(5)-\mathrm{C}(4)-\mathrm{H}(4)$ & 120.7 \\
\hline $\mathrm{C}(3)-\mathrm{C}(4)-\mathrm{H}(4)$ & 120.7 \\
\hline $\mathrm{N}(1)-\mathrm{C}(5)-\mathrm{C}(4)$ & $122.5(5)$ \\
\hline $\mathrm{N}(1)-\mathrm{C}(5)-\mathrm{H}(5)$ & 118.8 \\
\hline $\mathrm{C}(4)-\mathrm{C}(5)-\mathrm{H}(5)$ & 118.8 \\
\hline $\mathrm{C}(1)-\mathrm{C}(6)-\mathrm{N}(2)$ & $109.8(4)$ \\
\hline$C(1)-C(6)-H(6 A)$ & 109.7 \\
\hline $\mathrm{N}(2)-\mathrm{C}(6)-\mathrm{H}(6 \mathrm{~A})$ & 109.7 \\
\hline $\mathrm{C}(1)-\mathrm{C}(6)-\mathrm{H}(6 \mathrm{~B})$ & 109.7 \\
\hline $\mathrm{N}(2)-\mathrm{C}(6)-\mathrm{H}(6 \mathrm{~B})$ & 109.7 \\
\hline $\mathrm{H}(6 \mathrm{~A})-\mathrm{C}(6)-\mathrm{H}(6 \mathrm{~B})$ & 108.2 \\
\hline $\mathrm{N}(2)-\mathrm{C}(7)-\mathrm{C}(8)$ & $110.8(4)$ \\
\hline $\mathrm{N}(2)-\mathrm{C}(7)-\mathrm{C}(9)$ & $110.4(4)$ \\
\hline $\mathrm{C}(8)-\mathrm{C}(7)-\mathrm{C}(9)$ & $113.9(4)$ \\
\hline $\mathrm{N}(2)-\mathrm{C}(7)-\mathrm{H}(7)$ & 107.2 \\
\hline $\mathrm{C}(8)-\mathrm{C}(7)-\mathrm{H}(7)$ & 107.2 \\
\hline $\mathrm{C}(9)-\mathrm{C}(7)-\mathrm{H}(7)$ & 107.2 \\
\hline $\mathrm{C}(7)-\mathrm{C}(8)-\mathrm{H}(8 \mathrm{~A})$ & 109.5 \\
\hline $\mathrm{C}(7)-\mathrm{C}(8)-\mathrm{H}(8 \mathrm{~B})$ & 109.5 \\
\hline $\mathrm{H}(8 \mathrm{~A})-\mathrm{C}(8)-\mathrm{H}(8 \mathrm{~B})$ & 109.5 \\
\hline $\mathrm{C}(7)-\mathrm{C}(8)-\mathrm{H}(8 \mathrm{C})$ & 109.5 \\
\hline
\end{tabular}




\section{Continued}

\begin{tabular}{|c|c|}
\hline $\mathrm{H}(8 \mathrm{~A})-\mathrm{C}(8)-\mathrm{H}(8 \mathrm{C})$ & 109.5 \\
\hline $\mathrm{H}(8 \mathrm{~B})-\mathrm{C}(8)-\mathrm{H}(8 \mathrm{C})$ & 109.5 \\
\hline $\mathrm{O}(1)-\mathrm{C}(9)-\mathrm{O}(2)$ & $123.5(5)$ \\
\hline $\mathrm{O}(1)-\mathrm{C}(9)-\mathrm{C}(7)$ & $121.3(4)$ \\
\hline $\mathrm{O}(2)-\mathrm{C}(9)-\mathrm{C}(7)$ & $115.1(4)$ \\
\hline $\mathrm{N}(3)-\mathrm{C}(10)-\mathrm{C}(11)$ & $122.2(4)$ \\
\hline $\mathrm{N}(3)-\mathrm{C}(10)-\mathrm{C}(15)$ & $113.3(4)$ \\
\hline$C(11)-C(10)-C(15)$ & $124.5(4)$ \\
\hline$C(10)-C(11)-C(12)$ & $118.3(4)$ \\
\hline $\mathrm{C}(10)-\mathrm{C}(11)-\mathrm{H}(11)$ & 120.8 \\
\hline $\mathrm{C}(12)-\mathrm{C}(11)-\mathrm{H}(11)$ & 120.8 \\
\hline$C(11)-C(12)-C(13)$ & $119.6(4)$ \\
\hline $\mathrm{C}(11)-\mathrm{C}(12)-\mathrm{H}(12)$ & 120.2 \\
\hline $\mathrm{C}(13)-\mathrm{C}(12)-\mathrm{H}(12)$ & 120.2 \\
\hline$C(14)-C(13)-C(12)$ & $119.3(4)$ \\
\hline $\mathrm{C}(14)-\mathrm{C}(13)-\mathrm{H}(13)$ & 120.4 \\
\hline $\mathrm{C}(12)-\mathrm{C}(13)-\mathrm{H}(13)$ & 120.4 \\
\hline $\mathrm{N}(3)-\mathrm{C}(14)-\mathrm{C}(13)$ & $122.1(4)$ \\
\hline $\mathrm{N}(3)-\mathrm{C}(14)-\mathrm{H}(14)$ & 118.9 \\
\hline $\mathrm{C}(13)-\mathrm{C}(14)-\mathrm{H}(14)$ & 118.9 \\
\hline $\mathrm{N}(4)-\mathrm{C}(15)-\mathrm{C}(10)$ & $106.7(4)$ \\
\hline $\mathrm{N}(4)-\mathrm{C}(15)-\mathrm{H}(15 \mathrm{~A})$ & 110.4 \\
\hline $\mathrm{C}(10)-\mathrm{C}(15)-\mathrm{H}(15 \mathrm{~A})$ & 110.4 \\
\hline $\mathrm{N}(4)-\mathrm{C}(15)-\mathrm{H}(15 \mathrm{~B})$ & 110.4 \\
\hline $\mathrm{C}(10)-\mathrm{C}(15)-\mathrm{H}(15 \mathrm{~B})$ & 110.4 \\
\hline $\mathrm{H}(15 \mathrm{~A})-\mathrm{C}(15)-\mathrm{H}(15 \mathrm{~B})$ & 108.6 \\
\hline $\mathrm{N}(4)-\mathrm{C}(16)-\mathrm{C}(18)$ & $110.6(4)$ \\
\hline$N(4)-C(16)-C(17)$ & $110.9(4)$ \\
\hline$C(18)-C(16)-C(17)$ & $108.7(4)$ \\
\hline $\mathrm{N}(4)-\mathrm{C}(16)-\mathrm{H}(16)$ & 108.9 \\
\hline $\mathrm{C}(18)-\mathrm{C}(16)-\mathrm{H}(16)$ & 108.9 \\
\hline $\mathrm{C}(17)-\mathrm{C}(16)-\mathrm{H}(16)$ & 108.9 \\
\hline $\mathrm{C}(16)-\mathrm{C}(17)-\mathrm{H}(17 \mathrm{~A})$ & 109.5 \\
\hline $\mathrm{C}(16)-\mathrm{C}(17)-\mathrm{H}(17 \mathrm{~B})$ & 109.5 \\
\hline $\mathrm{H}(17 \mathrm{~A})-\mathrm{C}(17)-\mathrm{H}(17 \mathrm{~B})$ & 109.5 \\
\hline $\mathrm{C}(16)-\mathrm{C}(17)-\mathrm{H}(17 \mathrm{C})$ & 109.5 \\
\hline $\mathrm{H}(17 \mathrm{~A})-\mathrm{C}(17)-\mathrm{H}(17 \mathrm{C})$ & 109.5 \\
\hline $\mathrm{H}(17 \mathrm{~B})-\mathrm{C}(17)-\mathrm{H}(17 \mathrm{C})$ & 109.5 \\
\hline $\mathrm{O}(3)-\mathrm{C}(18)-\mathrm{O}(4)$ & $122.9(4)$ \\
\hline $\mathrm{O}(3)-\mathrm{C}(18)-\mathrm{C}(16)$ & $120.8(4)$ \\
\hline $\mathrm{O}(4)-\mathrm{C}(18)-\mathrm{C}(16)$ & $116.2(4)$ \\
\hline$N(5)-C(19)-S(1)$ & $178.9(4)$ \\
\hline $\mathrm{O}(2)-\mathrm{Co}(1)-\mathrm{O}(4)$ & $86.73(14)$ \\
\hline $\mathrm{O}(2)-\mathrm{Co}(1)-\mathrm{N}(3)$ & $91.93(13)$ \\
\hline
\end{tabular}




\section{Continued}

\begin{tabular}{|c|c|}
\hline $\mathrm{O}(4)-\mathrm{Co}(1)-\mathrm{N}(3)$ & $88.55(17)$ \\
\hline $\mathrm{O}(2)-\mathrm{Co}(1)-\mathrm{N}(1)$ & $93.53(13)$ \\
\hline $\mathrm{O}(4)-\mathrm{Co}(1)-\mathrm{N}(1)$ & $93.77(18)$ \\
\hline $\mathrm{N}(3)-\mathrm{Co}(1)-\mathrm{N}(1)$ & $174.18(16)$ \\
\hline $\mathrm{O}(2)-\mathrm{Co}(1)-\mathrm{N}(4)$ & $171.62(16)$ \\
\hline $\mathrm{O}(4)-\mathrm{Co}(1)-\mathrm{N}(4)$ & $86.88(17)$ \\
\hline $\mathrm{N}(3)-\mathrm{Co}(1)-\mathrm{N}(4)$ & $82.50(14)$ \\
\hline $\mathrm{N}(1)-\mathrm{Co}(1)-\mathrm{N}(4)$ & $92.29(14)$ \\
\hline $\mathrm{O}(2)-\mathrm{Co}(1)-\mathrm{N}(2)$ & $86.41(15)$ \\
\hline $\mathrm{O}(4)-\mathrm{Co}(1)-\mathrm{N}(2)$ & $172.66(16)$ \\
\hline $\mathrm{N}(3)-\mathrm{Co}(1)-\mathrm{N}(2)$ & $94.30(18)$ \\
\hline $\mathrm{N}(1)-\mathrm{Co}(1)-\mathrm{N}(2)$ & $84.03(18)$ \\
\hline $\mathrm{N}(4)-\mathrm{Co}(1)-\mathrm{N}(2)$ & $100.19(18)$ \\
\hline $\mathrm{C}(5)-\mathrm{N}(1)-\mathrm{C}(1)$ & $119.0(4)$ \\
\hline $\mathrm{C}(5)-\mathrm{N}(1)-\mathrm{Co}(1)$ & $126.0(4)$ \\
\hline $\mathrm{C}(1)-\mathrm{N}(1)-\mathrm{Co}(1)$ & $114.9(3)$ \\
\hline $\mathrm{C}(6)-\mathrm{N}(2)-\mathrm{C}(7)$ & $111.6(3)$ \\
\hline $\mathrm{C}(6)-\mathrm{N}(2)-\mathrm{Co}(1)$ & $108.6(3)$ \\
\hline $\mathrm{C}(7)-\mathrm{N}(2)-\mathrm{Co}(1)$ & $108.8(3)$ \\
\hline $\mathrm{C}(6)-\mathrm{N}(2)-\mathrm{H}(2 \mathrm{~A})$ & $108(3)$ \\
\hline $\mathrm{C}(7)-\mathrm{N}(2)-\mathrm{H}(2 \mathrm{~A})$ & $106(3)$ \\
\hline $\mathrm{Co}(1)-\mathrm{N}(2)-\mathrm{H}(2 \mathrm{~A})$ & $114(4)$ \\
\hline $\mathrm{C}(14)-\mathrm{N}(3)-\mathrm{C}(10)$ & $118.5(4)$ \\
\hline $\mathrm{C}(14)-\mathrm{N}(3)-\mathrm{Co}(1)$ & $126.1(3)$ \\
\hline $\mathrm{C}(10)-\mathrm{N}(3)-\mathrm{Co}(1)$ & $115.1(3)$ \\
\hline $\mathrm{C}(15)-\mathrm{N}(4)-\mathrm{C}(16)$ & $111.5(3)$ \\
\hline $\mathrm{C}(15)-\mathrm{N}(4)-\mathrm{Co}(1)$ & $107.5(2)$ \\
\hline $\mathrm{C}(16)-\mathrm{N}(4)-\mathrm{Co}(1)$ & $108.3(3)$ \\
\hline $\mathrm{C}(15)-\mathrm{N}(4)-\mathrm{H}(4 \mathrm{~A})$ & $106(3)$ \\
\hline $\mathrm{C}(16)-\mathrm{N}(4)-\mathrm{H}(4 \mathrm{~A})$ & $110(3)$ \\
\hline $\mathrm{Co}(1)-\mathrm{N}(4)-\mathrm{H}(4 \mathrm{~A})$ & $113(3)$ \\
\hline $\mathrm{C}(9)-\mathrm{O}(2)-\mathrm{Co}(1)$ & $116.0(3)$ \\
\hline $\mathrm{C}(18)-\mathrm{O}(4)-\mathrm{Co}(1)$ & $115.2(3)$ \\
\hline
\end{tabular}

Symmetry transformations used to generate equivalent atoms. 


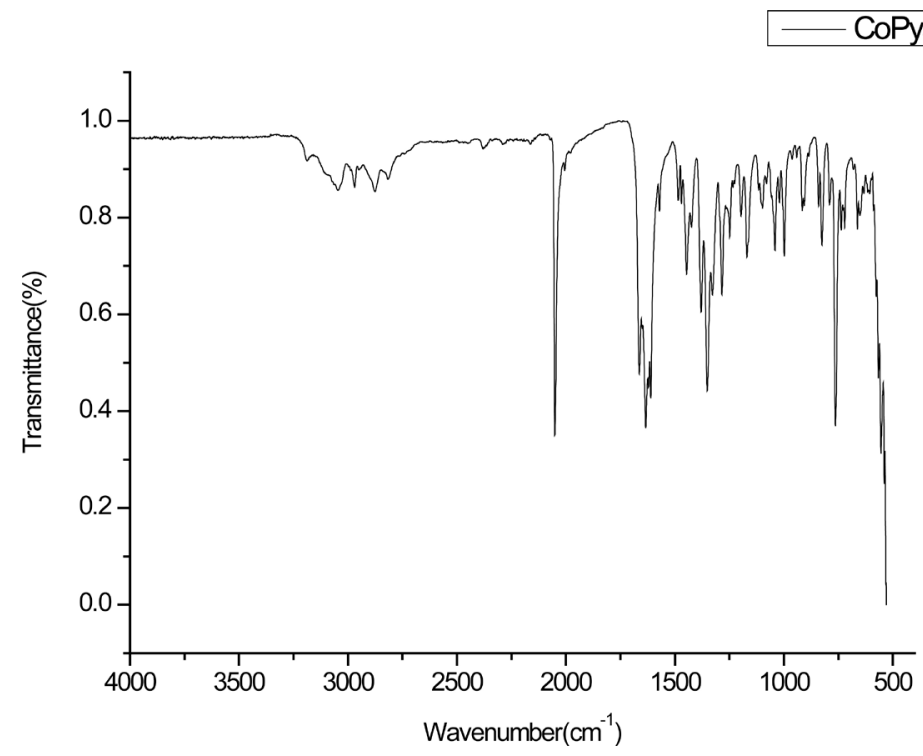

IR Spectrum.

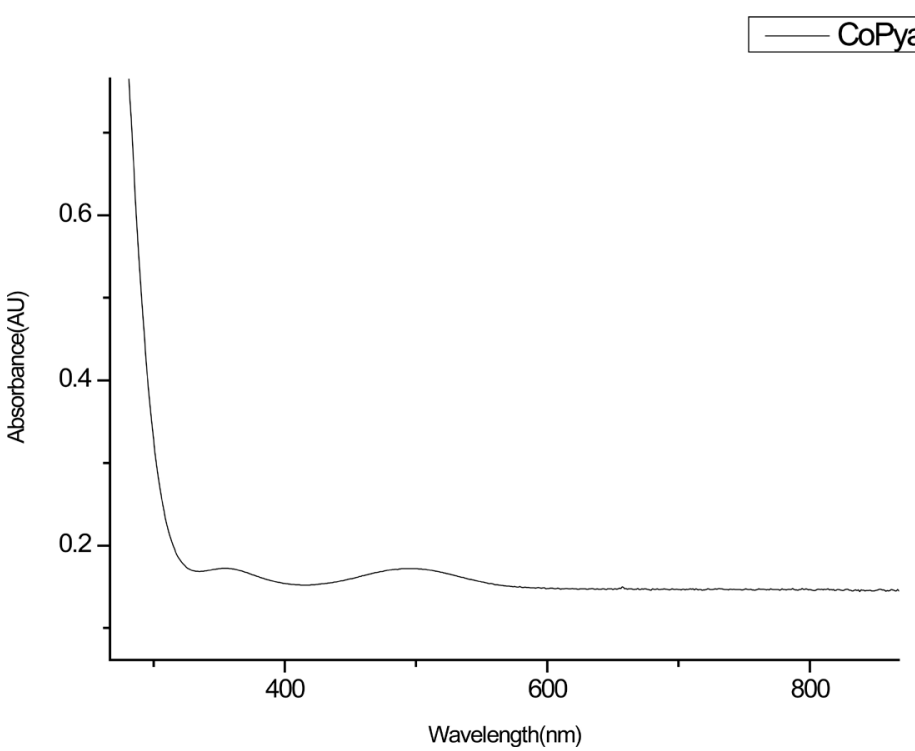

UV/Vis Spectrum.



M/Z Spectrum. 




TGA Spectrum.

Submit or recommend next manuscript to SCIRP and we will provide best service for you:

Accepting pre-submission inquiries through Email, Facebook, LinkedIn, Twitter, etc. A wide selection of journals (inclusive of 9 subjects, more than 200 journals)

Providing 24-hour high-quality service

User-friendly online submission system

Fair and swift peer-review system

Efficient typesetting and proofreading procedure

Display of the result of downloads and visits, as well as the number of cited articles

Maximum dissemination of your research work

Submit your manuscript at: http://papersubmission.scirp.org/

Or contactcsta@scirp.org 